\title{
Characterization of Colletotrichum spp. Sensitivity to Carbendazim for Isolates Causing Strawberry Anthracnose in China
}

\author{
Shan Zhong, ${ }^{1}$ Jianqiang Miao, ${ }^{2}$ Xili Liu, ${ }^{2}$ and Guozhen Zhang ${ }^{1, \dagger}$ \\ ${ }^{1}$ Department of Plant Pathology, College of Plant Protection, China Agricultural University, Beijing 100193, China \\ ${ }^{2}$ State Key Laboratory of Crop Stress Biology for Arid Areas, Northwest A\&F University, Yangling 712100, China
}

\begin{abstract}
Strawberry anthracnose caused by Colletotrichum species is an important disease that may cause significant economic losses. Based on multilocus sequence analyses and morphological characteristics, 64 isolates from strawberry anthracnose samples collected from nine Chinese provinces and municipalities were identified as three species: Colletotrichum fructicola (29 isolates), Colletotrichum siamense (23 isolates), and Colletotrichum nymphaeae (12 isolates). Isolates of $C$. siamense showed strong aggressiveness to fruit and leaves. Isolates of C. fructicola showed strong aggressiveness to crowns. Isolates of $C$. nymphaeae were weakly or not pathogenic to fruit, leaves, or crowns. Sensitivity to carbendazim was determined for a total of 75 isolates, including 11 previously preserved. Two isolates of $C$. siamense were highly re-

growth was not completely inhibited on potato dextrose agar amended with $500 \mu \mathrm{g} / \mathrm{ml}$ carbendazim. Beta-tubulin (TUB2) of representative isolates was amplified and sequenced, revealing a glutamic acid substituted by alanine at codon 198 in HR isolates of $C$. siamense and $C$. fructicola. MR isolates of $C$. siamense and $C$. fructicola had a point mutation at codon 200 , causing a replacement of phenylalanine acid by tyrosine. No point mutation was detected at codons 50, 167, 198, 200, or 240 in TUB2 of C. nymphaeae insensitive isolates. Overall, this study revealed that $C$. fructicola was the dominant species causing anthracnose on strawberry and could improve the understanding of the management of fungicide resistance in Colletotrichum species on strawberry in China.
\end{abstract} sistant (HR) and 21 were moderately resistant (MR). Nine isolates of C. fructicola were sensitive (S), 24 were HR, and four were MR. All 15 isolates of $C$. nymphaeae were insensitive, and their mycelial
Keywords: strawberry, Colletotrichum spp., phylogeny, aggressiveness, carbendazim
Strawberry (Fragaria $\times$ ananassa) is a major fruit crop in China, and China has been the largest strawberry producer in the world (Zhang et al. 2014). Growers in China plant $>129,700$ hectares and produced 3.42 million tons in 2016 (Ministry of Agriculture of the People's Republic of China 2018). Strawberry crown rot, caused by members of the genus Colletotrichum, remains one of the most severe disease problems in strawberry-producing areas in China, especially in greenhouses. The incidence of crown rot in seedlings reaches $\leq 80 \%$ in severe cases (Zhang 2015). Colletotrichum species can infect all plant parts, causing fruit rot, irregular spots on leaves, dark brown petiole and runner lesions, and crown rot. Pink or orange masses of spores on diseased tissues can be found during periods of high humidity and temperatures above $20^{\circ} \mathrm{C}$.

In China, strawberry anthracnose was reported for the first time in 1990, and the pathogen was identified as Colletotrichum fragariae (syn. Colletotrichum theobromicola; $\mathrm{Hu}$ 1990). Molecular tools are more reliable indicators for Colletotrichum species identification because of observations that the morphological features of Colletotrichum species can change with repeated subculturing or variable culture conditions (Johnston 2000). A comprehensive overview of the genus Colletotrichum was completed in 2009 and incorporated data generated through DNA analysis and phylogenetic systematics

${ }^{\dagger}$ Corresponding author: G. Zhang; zhanggzh@ cau.edu.cn

S. Zhong and J. Miao contributed equally to this work.

*The $\boldsymbol{e}$-Xtra logo stands for "electronic extra" and indicates that supplementary tables are published online.

The author(s) declare no conflict of interest.

Accepted for publication 26 June 2020.

(C) 2021 The American Phytopathological Society for the first time (Hyde et al. 2009). Morphological and molecular characteristics have since been combined to identify the pathogen of anthracnose at the species level (Damm et al. 2012; Weir et al. 2012). Based on morphological features and multigene phylogenetic analysis, Colletotrichum aenigma, Colletotrichum fructicola, Colletotrichum gloeosporioides, Colletotrichum murrayae (syn. Colletotrichum siamense; Liu et al. 2016), and Colletotrichum nymphaeae have been found on strawberry in Hubei, China (Han et al. 2016). C. fructicola and Colletotrichum viniferum have been detected in Shandong province (He et al. 2019). Colletotrichum changpingense, a new species, has been identified in Beijing (Jayawardena et al. 2016).

However, little is known of the species of Colletotrichum infecting strawberry in China. Previous limited studies focused on the Colletotrichum species that cause strawberry anthracnose were based on morphology and internal transcribed spacer (ITS) sequence data (Ren et al. 2008, 2011). By contrast, a few provinces identified species based on multigene phylogenetic and morphological studies (Han et al. 2016; He et al. 2019; Jayawardena et al. 2016). At present, there is no detailed research about isolates associated with strawberry anthracnose in China. Furthermore, sensitivity of these isolates to carbendazim and its mechanism were not clear. However, it is important for agricultural scientists and growers to improve the management of strawberry anthracnose.

Methyl benzimidazole carbamate (MBC) fungicides, including carbendazim, benomyl, and thiophanate-methyl, bind to betatubulin (TUB2) to inhibit spindle formation and prevent microtubule assembly, and subsequently they inhibit germ tube elongation and mycelial growth of the pathogen (Davidse 1986). MBCs have long been used to control anthracnose of numerous crops in China. Rapid development of MBC resistance of the $C$. gloeosporioides complex isolates associated with anthracnose of strawberry has arisen in recent years in Zhejiang and Hubei provinces (Han et al. 2018; Lin et al. 2016), limiting the continuous and widespread use of MBCs. Mutations in codons $6,50,167,198,200$, or 240 in TUB2 of fungal pathogens could lead to MBC fungicide resistance, and the most common point mutation occurs at codon 198 or 200 (Zhan et al. 2014). However, 
the prevalence of $\mathrm{MBC}$ resistance of Colletotrichum spp. infecting strawberry in China is unclear. Thus, the occurrence of carbendazim resistance in Colletotrichum spp. and the molecular mechanism of resistance in isolates causing strawberry anthracnose must be investigated.

Strawberry anthracnose is common in China, but a comprehensive understanding of the species of Colletotrichum and their resistance to MBCs is limited. Therefore, the aims of this study were to identify the causal agent and describe the species of Colletotrichum from nine provinces and municipalities in China between 2010 and 2017, based on morphological characteristics and multigene molecular phylogeny; assess the aggressiveness to strawberry of different Colletotrichum species; and evaluate isolate sensitivity to carbendazim and identify putative mutations in TUB2 for resistant isolates.

\section{Materials and Methods}

Fungal isolation. Sixty-four isolates of Colletotrichum spp. were obtained from infected strawberry samples collected from nine provinces and municipalities throughout China between 2010 and 2017. Diseased crowns, petiole, stolon, leaf, and fruit were soaked in $10 \mathrm{ml}$ of a $3.0 \% \mathrm{NaOCl}$ solution for $1 \mathrm{~min}$, washed with sterile distilled water three times, and subsequently plated on potato dextrose agar (PDA) (200 $\mathrm{g}$ of potato, $20 \mathrm{~g}$ of glucose, and $20 \mathrm{~g}$ of agar per liter) to obtain cultures. Cultures were incubated at $28^{\circ} \mathrm{C}$ in the dark for 3 to 5 days and then transferred to new PDA plates. Cultures were incubated on PDA $+0.1 \%$ yeast extract and mycelia growing 3 days were swabbed to promote sporulation for cultures that do not sporulate on PDA (Huang and Zhang 2014). A germinated single spore was transferred to PDA for purification and incubated at $28^{\circ} \mathrm{C}$.

Morphological characteristics. Mycelial plugs were transferred from the edge of active colonies onto fresh PDA plates and incubated at $28^{\circ} \mathrm{C}$ in the dark. The morphological characteristics of each isolate were evaluated, including colony, conidial, and appressorial characteristics. Colony characteristics were determined after incubation for 10 days. The colony characteristics of five representative isolates of each species were recorded, with the lengths and widths of 30 randomly chosen conidia measured. Appressoria were induced using slide culture technology (Johnston and Jones 1997), and the shape and color of appressoria were recorded.

DNA extraction, PCR amplification, and sequencing. Mycelia were collected from cultures grown on PDA. Genomic DNA was extracted from all isolates by the modified method according to Damm et al. (2008). PCR amplification was performed for six loci: the ITS regions, glyceraldehyde-3-phosphate dehydrogenase $(G A P D H)$, chitin synthase $1(C H S-1)$, actin $(A C T), T U B 2$, and histone 3 (HIS3) (for the Colletotrichum acutatum complex) or calmodulin $(C A L)$ (for the $C$. gloeosporioides complex). The primers used in this study are listed in Table 1 . The amplified products of each gene were separated on agarose gel $(1.0 \% \mathrm{wt} / \mathrm{vol})$ by electrophoresis and sequenced by TSINGKE, Beijing, China, with a 3730 DNA analyzer.

Sequence alignment and molecular identification. Multiple sequence alignments were generated in MAFFT (https://mafft.cbrc.jp/ alignment/server/index.html; Kuraku et al. 2013) and manually edited in BioEdit version 7.1.11 sequence alignment editing software (Hall 1999). Bayesian analyses were performed on consensus sequences in MrBayes version 3.2.2 software (Ronquist et al. 2012). Nucleotide substitution models were selected with MrModeltest version 2.3 software (Nylander 2004).

Six loci (ACT, CAL, CHS-1, GAPDH, ITS, and TUB2) were used to establish a concatenated dataset of representative isolates including 81 fungal taxa based on morphological characteristics of the $C$. gloeosporioides complex. Additionally, six loci (ITS, GAPDH, $C H S-1, H I S 3, A C T$, and TUB2) were used for multilocus analysis of representative isolates including 58 fungal taxa, based on morphological characteristics of the C. acutatum complex. The sequences of reference isolates and outgroups Colletotrichum boninense (for the C. gloeosporioides complex) and Colletotrichum orchidophilum (for the C. acutatum complex) obtained from GenBank for phylogenetic analysis are listed in Supplementary Tables S1 and S2.

Pathogenicity tests. Three representative isolates of each species were used in pathogenicity tests repeated twice to test the pathogenicity of the three species. All representative isolates of $C$. fructicola and $C$. siamense originally came from diseased crowns, and one representative isolate of $C$. nymphaeae came from a stolon and two came from diseased petioles. Asymptomatic seedlings and detached fully developed leaves of Benihoppe strawberry and detached fruits of Poinsettia strawberry of the same age were used in the pathogenicity tests. Five fruits, 15 leaves, and three seedlings per isolate were inoculated.

Detached fruits were soaked in $70 \%$ ethanol for $30 \mathrm{~s}$, and leaves were sprayed with $70 \%$ ethanol until the leaf surface was covered and then the leaves were washed with sterile distilled water three times. The concentration of the conidia suspension was adjusted to $1 \times 10^{5}$ spores per milliliter for inoculation of the detached fruit and leaves. Fruit and leaves were inoculated with sterile water to serve as controls. Fruits were wounded $3 \mathrm{~mm}$ deep with a sterile dissecting needle inoculated with $10 \mu \mathrm{l}$ of conidia suspension, then incubated at $28^{\circ} \mathrm{C}$ in the dark for $24 \mathrm{~h}$ and then in $12 \mathrm{~h}$ light $/ 12 \mathrm{~h}$ dark for $48 \mathrm{~h}$ with $80 \%$ relative humidity. The aggressiveness to the fruits was measured by the diameter of lesions. Leaves were sprayed with the conidia suspension until the leaf surface was covered with drops, then incubated at $28^{\circ} \mathrm{C}$ in the dark for $24 \mathrm{~h}$ and then in $12 \mathrm{~h}$ light $/ 12 \mathrm{~h}$ dark for 6 days with $80 \%$ relative humidity. The aggressiveness to leaves of the three species was measured bydisease severity. Disease severity was scored on a 0 - to 9-point scale based on the percentage infected area, and the scale of the severity of disease was converted to the disease index described by Wang et al. (2008). In brief, $0=$ healthy leaves with no lesions, $1=$ leaves with small black spots and percentage infected area $<5 \%, 3=$ leaves with round or oval lesions accounting for 5.1 to $15 \%, 5=$ leaves with confluent large lesions accounting for 15.1 to $30 \%, 7=$ leaves with large black lesions accounting for 30.1 to $50 \%$, and $9=$ dead leaves or percentage infected area from 50.1 to $100 \%$. Disease index $=\left[\left(n_{0} \times 0+n_{1}\right.\right.$

Table 1. Primers used in this study, with sequences and sources

\begin{tabular}{llll}
\hline Gene & Primer & \multicolumn{1}{c}{ Sequence (5' ${ }^{\prime} \mathbf{3}^{\prime}$ ) } & Reference \\
\hline ACT & ACT-512F & ATGTGCAAGGCCGGTTTCGC & Carbone and Kohn 1999 \\
& ACT-783R & TACGAGTCCTTCTGOCCCAT & Carbone and Kohn 1999 \\
CAL & CL1C & GAATTCAAGGAGGCCTTCTC & Weir et al. 2012 \\
& CL2C & CTTCTGCATCATGAGCTGGAC & Weir et al. 2012 \\
CHS-1 & CHS-79F & TGGGGCAAGGATGCTTGGAAGAAG & Carbone and Kohn 1999 \\
& CHS-354R & TGGAAGAACCATCTGTGAGAGTTG & Carbone and Kohn 1999 \\
GAPDH & GDF1 & GCCGTCAACGACCCCTTCATTGA & Guerber et al. 2003 \\
& GDR1 & GGGTGGAGTCGTACTTGAGCATGT & Guerber et al. 2003 \\
HIS3 & CYLH3F & AGGTCCACTGGTGGCAAG & Crous et al. 2004 \\
& CYLH3R & AGCTGGATGTCCTTGGACTG & Crous et al. 2004 \\
ITS & ITS 1 & TCCGTAGGTGAACCTGCGG & White et al. 1990 \\
& ITS 4 & TCCTCCGCTTATTGATATGC & White et al. 1990 \\
TUB2 & T1 & AACATGCGTGAGATTGTAAGT & Glass and Donaldson 1995 \\
& Bt2b & ACCCTCAGTGTAGTGACCCTTGGC & O'Donnell and Cigelnik 1997 \\
\hline
\end{tabular}


$\left.\left.\times 1+n_{3} \times 3+n_{5} \times 5+n_{7} \times 7+n_{9} \times 9\right) / 9 N\right] \times 100$. The numbers $0,1,3$, 5,7 , and $9=$ point scale, $n=$ the number of leaves in its scale, and $N=$ the total number of leaves. Crowns were exposed by digging out soil on the side of the seedlings. A cross-wound $(5 \mathrm{~mm} \times 10 \mathrm{~mm})$ was made on the crowns with a sterile scalpel and inoculated with mycelial plugs $(5 \mathrm{~mm} \times 10 \mathrm{~mm})$ cut from the edge of a 5-day-old colony on PDA. Injured crowns were treated with PDA plugs $(5 \mathrm{~mm} \times 10 \mathrm{~mm})$ alone as controls and covered with soil at $28^{\circ} \mathrm{C}$ in $12 \mathrm{~h}$ light $/ 12 \mathrm{~h}$ dark for 20 days with $80 \%$ relative humidity. The aggressiveness to crowns of the three species was measured by disease severity. The severity of disease was scored on a 0 - to 4-point scale based on the percentage infected area of the crown longitudinal section, and the scale of the severity of disease was converted to the disease index described by Jin (2013). In brief, $0=$ healthy crown with no lesions, $1=$ crown with light lesions and percentage infected area $<25 \%, 2=$ lesions account for 25.1 to $50 \%, 3=$ lesions account for 50.1 to $75 \%$, and $4=$ lesions account for 75.1 to $100 \%$ and seedlings are extremely weak or dead. Disease index $=\left[\left(n_{0} \times 0+n_{1} \times 1+n_{2} \times 2+n_{3} \times 3+n_{4} \times\right.\right.$ $4) / 4 N] \times 100$. The numbers $0,1,2,3$, and $4=$ point scale, $n=$ the number of crowns in its scale, and $N=$ the total number of crowns.

Fungicide sensitivity assays. The carbendazim (98.0\% active ingredient) was kindly provided by Anhui Guangxin Agrochemical Co., Ltd., China. A $10^{4} \mathrm{mg} / \mathrm{ml}$ stock solution was made in hydrochloric acid ( $\mathrm{HCl} ; 0.1 \mathrm{~mol} /$ liter). The sensitivity of the 75 isolates (including 11 isolates previously preserved in our lab) to carbendazim of six concentrations $(0,0.1,1,10,100$, and $500 \mu \mathrm{g} / \mathrm{ml})$ was determined in vitro using the mycelia growth assay. The evaluation grouped resistance levels into four representative phenotypes by reaction as described in previous studies (Baggio et al. 2018; Nalumpang et al. 2010; Peres et al. 2004): highly resistant (HR; isolate grew normally on PDA amended with $500 \mu \mathrm{g} / \mathrm{ml}$ of carbendazim), moderately resistant (MR; isolate grew on PDA amended with $100 \mu \mathrm{g} / \mathrm{ml}$ of carbendazim), sensitive (S, isolate grew on PDA amended with $\leq 1 \mu \mathrm{g} / \mathrm{ml}$ of carbendazim), and insensitive (IS, isolate shows the same inhibition of mycelial growth on PDA amended with $\geq 10 \mu \mathrm{g} / \mathrm{ml}$ of carbendazim, and growth of isolate is not completely inhibited because it grew slowly with continued incubation). Each treatment was incubated at $28^{\circ} \mathrm{C}$ for 3 days, repeated twice for three replicate plates.

Amplification and sequencing of $\boldsymbol{T U B 2}$ genes. To determine whether common mutations associated with resistance to carbendazim at codons 198 and 200 of TUB2 were present, the primer pair TUBGF1/TUBGR was used for representative isolates of the $C$. gloeosporioides complex (Chung et al. 2010). The primer pair CaTUB2-upT/CaTUB2-doT was used for representative isolates of the $C$. acutatum complex to amplify a fragment of TUB2 carrying codons 50, 167, 198, 200, and 240 (Nakaune and Nakano 2007). DNAMAN version 5.2.2.0 software was used for the sequence analysis.

\section{Results}

Fungal isolation and morphological characteristics. A total of 64 isolates of Colletotrichum spp. were collected from infected strawberry samples from nine provinces and municipalities throughout China between 2010 and 2017. Thirty-eight strains (59.4\%) were isolated from crown, three $(4.7 \%)$ from fruits, seven (10.9\%) from leaves, nine (14.1\%) from petioles, and seven (10.9\%) from stolons. Information on the geographic location of isolate sample collection is detailed in Table 2.

Based on the colony and conidial morphology on PDA, all strains were assigned to three groups. Groups I and II contained 29 and 23 strains, respectively; those with cylindrical conidia with round ends were assigned to the $C$. gloeosporioides species complex. Group III contained 12 strains producing fusiform conidia, assigned to the C. acutatum species complex.

Colonies of group I were flat, with the entire edge on the PDA, olive to gray in the center with a white edge. The aerial mycelium was pale white and cottony (Fig. 1a), with the reverse grayish green to dark gray in the center with a white halo (Fig. 1b). Conidia were hyaline and cylindrical, with both ends rounded (Fig. 1c), measuring 10 to $12.8 \times 4$ to $5.9 \mu \mathrm{m}$. Appressoria were brown to dark brown, ovoid to elliptical or irregular in outline, undulate to lobate in margin, and solitary (Fig. 1d to 1f).

Colonies of group II were white and became light gray at the center with age. The aerial mycelium was pale white, cottony, and dense. Acervuli had a visible orange conidial mass at the inoculum point (Fig. 1g). The reverse side was gray near the center (Fig. 1h). Conidia were hyaline and cylindrical, with both ends rounded, measuring 10.5 to $13.5 \times 4.5$ to $5.5 \mu \mathrm{m}$ (Fig. 1i). Appressoria were medium to dark brown, ovoid to ellipsoid, lobed or irregular, and solitary (Fig. $1 \mathrm{j}$ to 11 ).

Colonies of group III were white to light gray. The aerial mycelium was white (Fig. 1m), and the reverse side was light gray to pale ocherous (Fig. 1n). Conidia were hyaline and fusiform, with both ends acute (Fig. 1o), measuring 10.1 to $14.7 \times 3.4$ to $4.7 \mu \mathrm{m}$. Appressoria were medium brown, elliptical or irregular, and solitary (Fig. 1p and $1 \mathrm{q})$.

Phylogenetic analysis. Based on morphological features, nine representative isolates from group I, 11 isolates from group II, and three representative isolates from group III were selected for multilocus phylogenetic analyses. For the $C$. gloeosporioides complex, the Bayesian inference related to isolates in this study clustered in two clades. Among nine isolates clustered with $C$. fructicola, seven clustered with ICMP18613 and ICMP18727, two clustered with CBS238.49 and CBS125395, and 11 clustered with $C$. siamense (ICMP12567, CBS125378, GZAAS5.09506, CBS130420, and ICMP18121) (Weir et al. 2012); the Bayesian posterior probabilities were all 0.99 (Fig. 2). For the C. acutatum complex, isolates clustered with $C$. nymphaeae (CBS100065, CBS122111, CBS125961, CBS127608, CBS129336, and CBS113002) (Damm et al. 2012), the Bayesian posterior probabilities were 0.99 (Fig. 3). Based on the multilocus phylogenies and morphological characteristics, 29 isolates were identified as $C$. fructicola, 23 were identified as $C$. siamense, and 12 were identified as $C$. nymphaeae (Table 2).

Aggressiveness assay. All isolates of the three species tested had pathogenicity to fruits, leaves, and crowns. Fruits inoculated with isolates of three species showed varying degrees of symptoms after 3 days. The diameters of the lesions caused by $C$. fructicola, $C$. siamense, and $C$. nymphaeae were $13.2 \mathrm{~mm}, 20.0 \mathrm{~mm}$, and $9.0 \mathrm{~mm}$, respectively. No symptoms were observed in the control fruits. Fruits

Table 2. Isolates of Colletotrichum spp. identified in this study with details about location, host tissues, and number of isolates

\begin{tabular}{|c|c|c|c|}
\hline Location & Species & Host tissue & Number of isolates \\
\hline \multirow[t]{3}{*}{ Anhui } & C. fructicola & Leaf & 1 \\
\hline & C. siamense & Crown & 2 \\
\hline & & Petiole & 1 \\
\hline \multirow[t]{11}{*}{ Beijing } & C. fructicola & Crown & 15 \\
\hline & & Leaf & 1 \\
\hline & & Fruit & 2 \\
\hline & & Stolon & 1 \\
\hline & C. nymphaeae & Crown & 1 \\
\hline & & Leaf & 2 \\
\hline & & Petiole & 4 \\
\hline & & Stolon & 3 \\
\hline & C. siamense & Crown & 10 \\
\hline & & Leaf & 3 \\
\hline & & Petiole & 1 \\
\hline Guangdong & C. siamense & Crown & 1 \\
\hline Guangxi & C. siamense & Petiole & 1 \\
\hline \multirow[t]{4}{*}{ Hebei } & C. nymphaeae & Petiole & 1 \\
\hline & & Fruit & 1 \\
\hline & C. siamense & Crown & 2 \\
\hline & & Stolon & 2 \\
\hline Liaoning & C. fructicola & Crown & 1 \\
\hline \multirow[t]{3}{*}{ Shandong } & C. fructicola & Crown & 3 \\
\hline & & Petiole & 1 \\
\hline & & Stolon & 1 \\
\hline Shanghai & C. fructicola & Crown & 1 \\
\hline Zhejiang & C. fructicola & Crown & 2 \\
\hline
\end{tabular}


inoculated with isolates of $C$. siamense showed the highest disease severity, producing large rotted lesions and orange conidial masses on the fruit surface (Fig. 4b). Fruits inoculated with isolates of $C$. fructicola developed dark brown decay (Fig. 4a). The aggressiveness of isolates of $C$. nymphaeae was the lowest; the infected fruits showed small sunken lesions covered with white aerial mycelia (Fig. 4c).

The disease indexes of leaves infected by $C$. fructicola, $C$. siamense, and $C$. nymphaeae were 72.3, 93.6, and 3.2, respectively, after 7 days' inoculation. No symptoms were observed in the control leaves. Leaves inoculated with isolates of $C$. siamense developed large, dark brown to black lesions from a confluence of small lesions (Fig. 4e), suggesting that isolates of $C$. siamense showed the strongest aggressiveness on leaves. Fewer black lesions occurred on leaves inoculated with isolates of $C$. fructicola than with isolates of C. siamense (Fig. 4d). Isolates of C. fructicola were found to be moderately aggressive in the capacity to cause anthracnose lesions on leaves. Small black spots were produced on leaves inoculated with C. nymphaeae, reflecting the weakest aggressiveness on leaves (Fig. 4f).

Strawberry seedlings, which were inoculated with isolates of $C$. fructicola and $C$. siamense, showed wilting after 20 days. No symptoms were observed in the seedlings inoculated with isolates of $C$. nymphaeae and noninoculated controls. The disease indexes of infected crowns caused by $C$. fructicola, C. siamense, and C. nymphaeae were $91.7,63.9$, and 8.3 , respectively. The seedlings inoculated with isolates of $C$. fructicola and $C$. siamense wilted and the longitudinal section of infected crowns showed reddish brown to dark brown lesions (Fig. 4g, 4h). No obvious discoloration was observed in the crowns inoculated with $C$. nymphaeae. The disease index indicated that isolates of $C$. fructicola showed the strongest aggressiveness, and $C$. siamense could infect crowns in a moderately strong manner, whereas isolates of $C$. nymphaeae showed the weakest crown aggressiveness (Fig. 4i).

Carbendazim sensitivity evaluation. The 64 isolates in this study and 11 isolates previously preserved in our lab from three species were used to screen sensitivity to carbendazim. Of $23 \mathrm{C}$. siamense isolates, $8.7 \%$ (two isolates) were HR and 91.3\% (21 isolates) were MR. Of 37 C. fructicola isolates, $24.3 \%$ (nine isolates) were $\mathrm{S}, 10.8 \%$ (four isolates) were MR, and 64.9\% (24 isolates) were HR. Of $15 C$. nymphaeae isolates, $100 \%$ were IS to carbendazim (Table 3 ).

Analysis of TUB2 gene from sensitive and resistant Colletotrichum spp. For the $C$. gloeosporioides complex species, $T U B 2$ gene sequences of wild-type isolates of $C$. gloeosporioides from GenBank (accession number U14138) and the representative isolates in this study were translated and analyzed. Compared with the wildtype isolates, no point mutation was detected in the sensitive isolates of $C$. fructicola. The MR phenotype of $C$. fructicola and $C$. siamense revealed one mutation in codon 200 and resulted in the amino acid (codon) phenylalanine (TTC) being replaced by tyrosine (TAC). The HR phenotype of $C$. fructicola and $C$. siamense revealed one mutation in codon 198 and resulted in the amino acid (codon) glutamic acid (GAG) being replaced by alanine (GCG). For the $C$. acutatum complex species, TUB2 gene sequences of wild-type isolates of $C$. acutatum (accession number AB273716) and representative isolates of $C$. nymphaeae that were insensitive to carbendazim in this study were translated and analyzed. No mutation was found in codons 50, 167, 198, 200 , or 240 (Table 4).
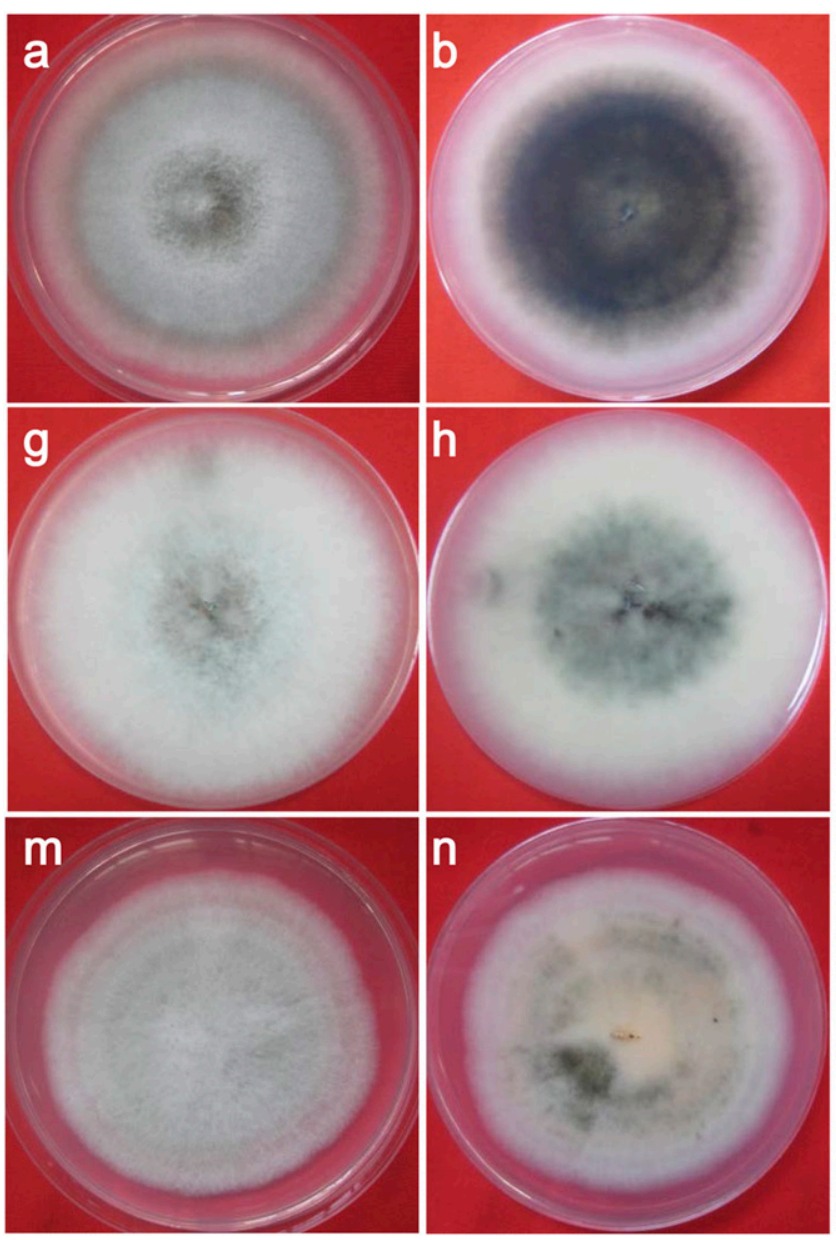
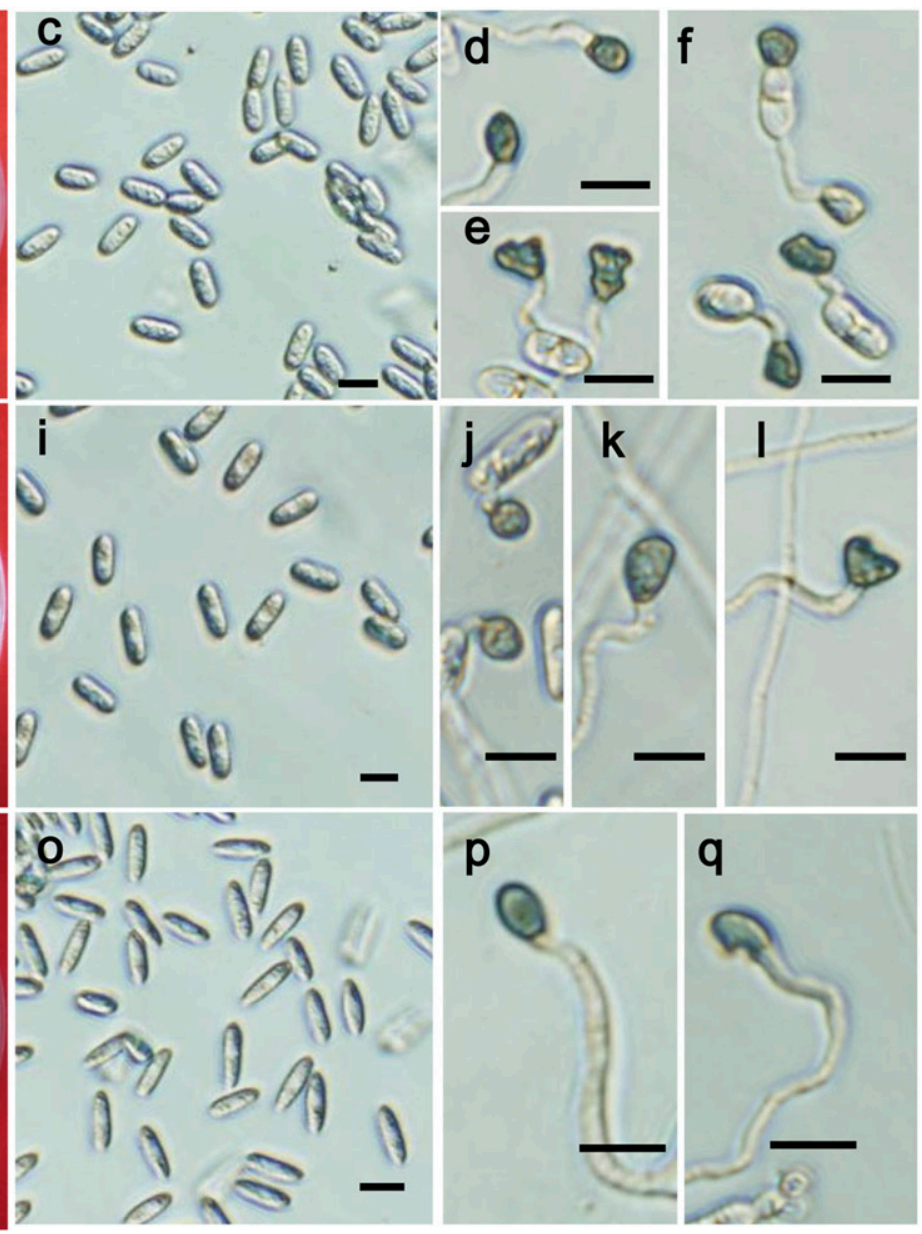

Fig. 1. Colonial, conidial, and appressorial morphological characters of Colletotrichum spp. from strawberry anthracnose samples in China. a-f, Group I. g-l, Group II. $m-\mathbf{q}$, Group III. a, g, m, Upper side of colony on PDA after incubation at $28^{\circ} \mathrm{C}$ for 7 days. b, h, $\mathrm{n}$, Reverse side of colony on PDA after incubation at $28^{\circ} \mathrm{C}$ for 7 days. c, i, o, Conidia (on PDA + $0.1 \%$ yeast extract). $\mathbf{d}-\mathbf{f}, \mathbf{j}-\mathbf{l}, \mathbf{p}, \mathbf{q}$, Appressoria (on glass slide). Bars $=10 \mu \mathrm{m}$. 


\section{Discussion}

This study identified isolates of Colletotrichum infecting strawberry collected from multiple provinces and municipalities throughout China, based on morphological and cultural characterization and multilocus phylogenetic analyses. Isolates of Colletotrichum were allocated to two species complexes and further assigned to three species.
C. fructicola was the dominant Colletotrichum species on strawberry, comprising $49.3 \%$ of all isolates, followed by C. siamense at $30.7 \%$ and C. nymphaeae at $20.0 \%$.

Species such as C. aenigma, C. fructicola, C. gloeosporioides, C. murrayae (syn C. siamense; Liu et al. 2016), C. nymphaeae, and $C$. viniferum have recently been reported in Hubei and Shandong

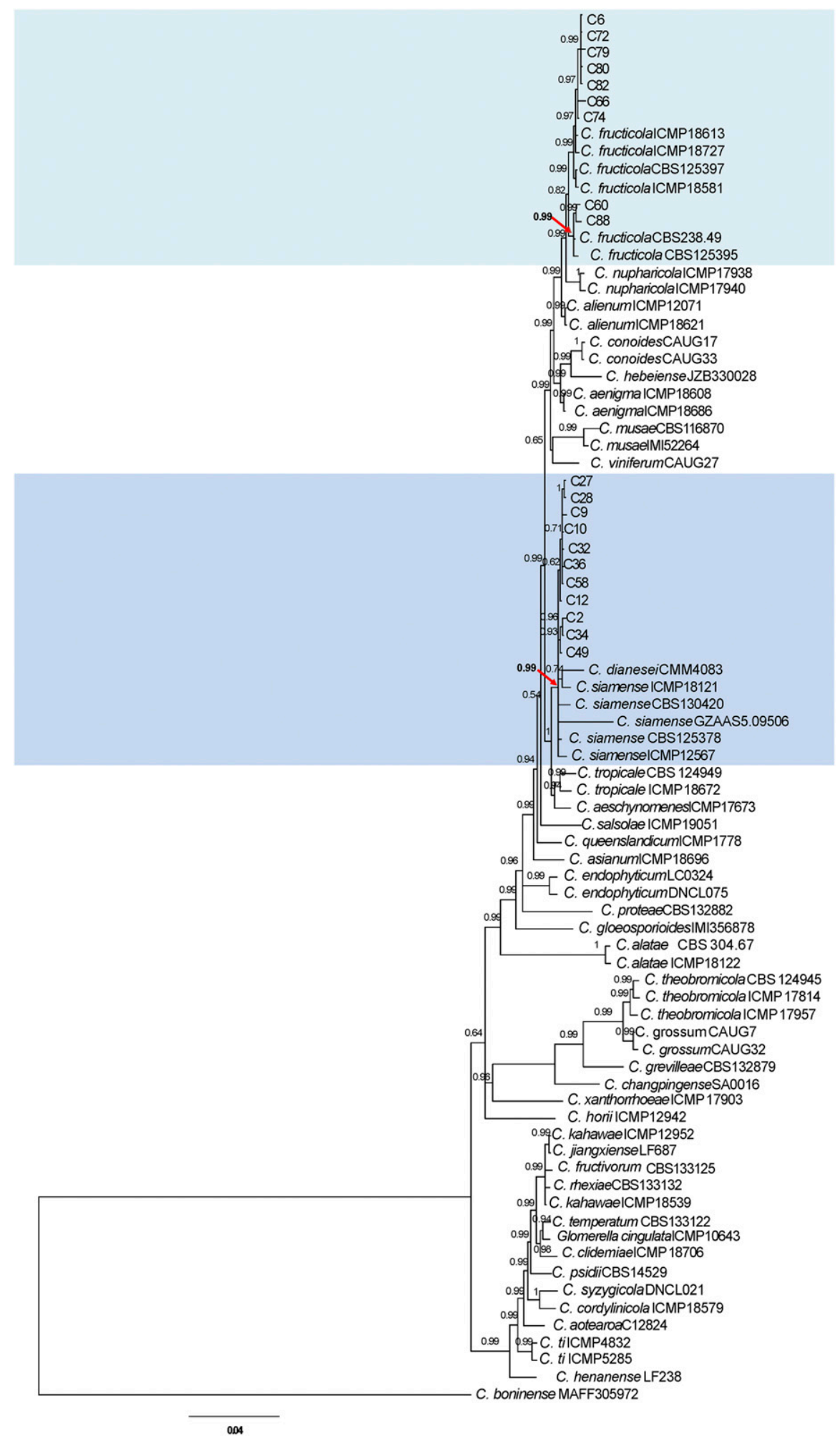

Fig. 2. Molecular phylogeny of the $C$. gloeosporioides complex and outgroups $C$. boninense presented in a Bayesian inference phylogenetic tree inferred from the dataset containing partial DNA sequences of ACT, CAL, CHS-1, GAPDH, ITS, and TUB2. 
provinces, according to morphological characteristics and phylogenetic analyses based on multilocus sequence data, isolated mainly from aboveground parts of strawberry such as leaves, fruit, and stolon (Han et al. 2016; He et al. 2019). Crown rot is of particular concern to the Chinese strawberry industry, and isolates of $C$. fructicola and $C$. siamense made up the majority of these crown rot isolates, but only one isolate of C. nymphaeae obtained from crowns. Other species such as C. aenigma, $C$. gloeosporioides, and $C$. viniferum were not found in the crown isolate collection, which is a possible artifact of the sample size.

The three species identified in this study showed different aggressiveness to strawberry. The disease indexes of crowns infected by $C$. fructicola, C. siamense, and C. nymphaeae were 91.7, 63.9, and 8.3, respectively, which showed that isolates of $C$. fructicola were the

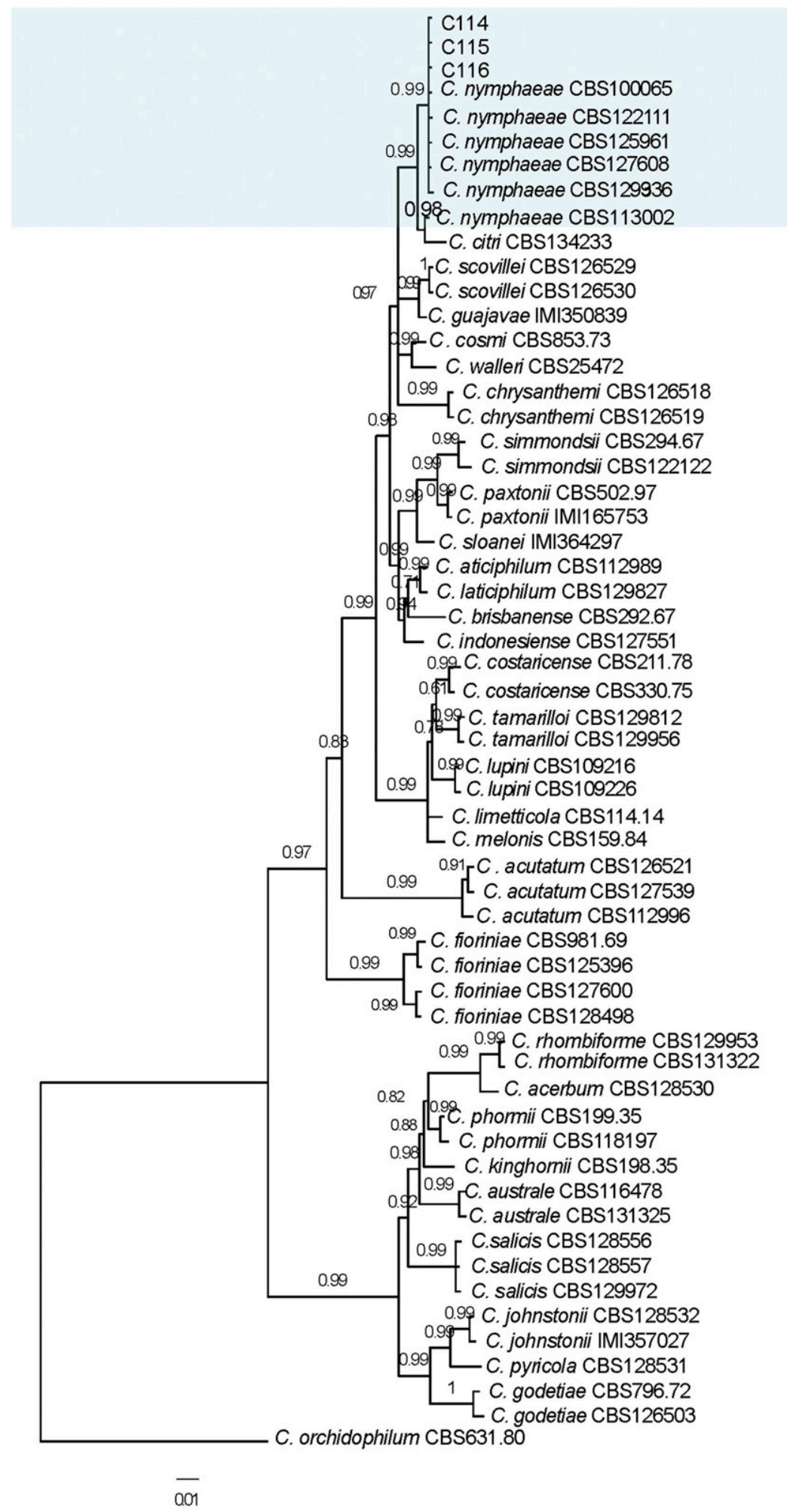

Fig. 3. Molecular phylogeny of the $C$. acutatum complex and outgroups $C$. orchidophilum presented in a Bayesian inference phylogenetic tree inferred from the dataset containing partial DNA sequences of ITS, GAPDH, CHS-1, HIS3, ACT, and TUB2. 

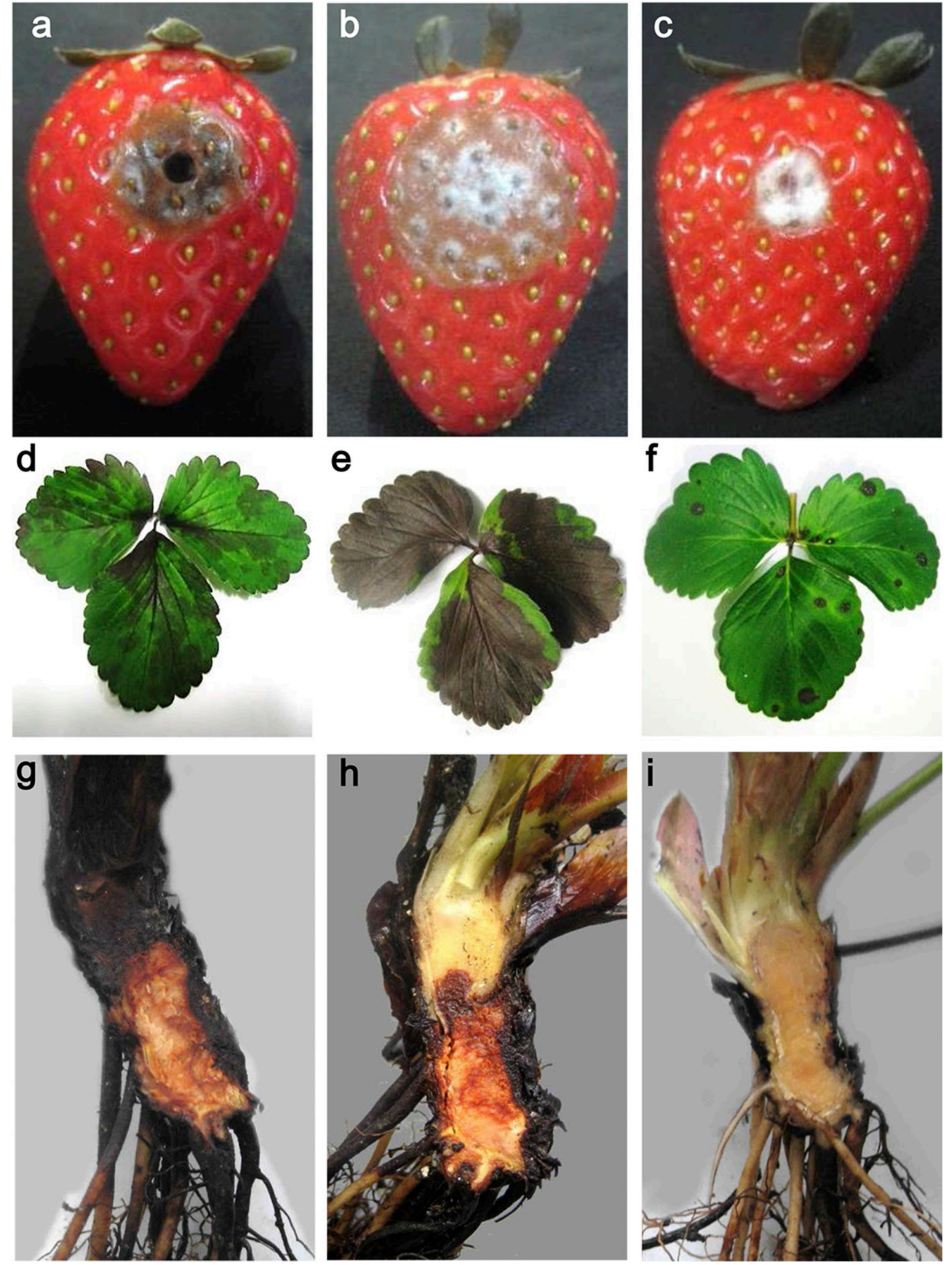

Fig. 4. Symptoms on strawberry caused by three Colletotrichum species. a-c, Strawberry fruits inoculated with $C$. fructicola, $C$. siamense, and $C$. nymphaeae, respectively, after 3 days. $\mathbf{d}-\mathbf{f}$, Strawberry leaves inoculated with $\mathrm{C}$. fructicola, C. siamense, and C. nymphaeae, respectively, after 7 days. $\mathbf{g}-\mathbf{i}$, Strawberry crowns inoculated with $\mathbf{C}$. fructicola, $C$. siamense, and $\mathrm{C}$. nymphaeae, respectively, after 20 days. 
most aggressive to crowns. The lesion size on fruit caused by $C$. siamense was $20.0 \mathrm{~mm}$, which was the largest compared with the lesion size caused by $C$. fructicola and $C$. nymphaeae. Furthermore, the disease indexes on leaves infected by $C$. fructicola, $C$. siamense, and $C$. nymphaeae were $72.3,93.6$, and 3.2 , respectively, which suggested that isolates of $C$. siamense showed the strongest aggressiveness on fruit and leaves. Isolates of C. nymphaeae showed weak aggressiveness to fruit and leaves and were even nonpathogenic to strawberry crowns, which was the same as Han described in Hubei province (Han et al. 2016), suggesting that the low isolation frequency of $C$. nymphaeae was caused by its weak aggressiveness. The evidence of isolation of the three species in strawberry tissues and their aggressiveness to different strawberry parts suggest that $C$. fructicola was the dominant species infecting strawberry crowns.

For the C. gloeosporioides complex, most isolates in the study showed resistance to carbendazim, with a resistance frequency of 85\%. Similar observations occurred in Zhejiang, where $98.2 \%$ of C. gloeosporioides complex species isolates from diseased strawberry tissues were resistant to MBCs (Lin et al. 2016). The median effective concentration for isolates of the $C$. gloeosporioides

Table 3. Percentage of different carbendazim phenotypes of three Colletotrichum species

\begin{tabular}{lcccc}
\hline & \multicolumn{4}{c}{ Carbendazim phenotype $^{\mathbf{a}}$} \\
\cline { 2 - 5 } Species & HR & MR & S & IS \\
\hline C. fructicola & $64.9 \%$ & $10.8 \%$ & $24.3 \%$ & $-{ }^{\mathrm{b}}$ \\
C. siamense & $8.7 \%$ & $91.3 \%$ & 0 & - \\
C. nymphaeae & - & - & - & $100 \%$ \\
\hline
\end{tabular}

${ }^{\text {a }}$ Carbendazim phenotypes of three Colletotrichum species associated with strawberry. HR, MR, S, and IS represent highly resistant, moderately resistant, sensitive, and insensitive, respectively.

$\mathrm{b}$ Dashes indicate the species did not have this carbendazim phenotype.

Table 4. Point mutations in the beta-tubulin gene (TUB2) of the three Colletotrichum species associated with strawberry exhibiting different phenotypes to carbendazim

\begin{tabular}{llll}
\hline Species & Isolate $^{\mathbf{a}}$ & Phenotype $^{\mathbf{b}}$ & Amino acid (codon) substitution \\
\hline C. fructicola & C38 & HR & E198A (GAG to GCG) \\
& C53 & HR & E198A (GAG to GCG) \\
& C54 & HR & E198A (GAG to GCG) \\
& C55 & HR & E198A (GAG to GCG) \\
& C64 & HR & E198A (GAG to GCG) \\
& C72 & HR & E198A (GAG to GCG) \\
& C74 & HR & E198A (GAG to GCG) \\
& C81 & HR & E198A (GAG to GCG) \\
& C83 & HR & E198A (GAG to GCG) \\
& C39 & MR & F200Y (TTC to TAC) \\
& C3 & S & None \\
C. siamense & C49 & HR & E198A (GAG to GCG) \\
& C2 & MR & F200Y (TTC to TAC) \\
& C12 & MR & F200Y (TTC to TAC) \\
& C28 & MR & F200Y (TTC to TAC) \\
& C32 & MR & F200Y (TTC to TAC) \\
& C34 & MR & F200Y (TTC to TAC) \\
& C45 & MR & F200Y (TTC to TAC) \\
& C47 & MR & F200Y (TTC to TAC) \\
C20 & IS & None \\
C24 & IS & None \\
C41 & IS & None \\
C69 & IS & None \\
\hline
\end{tabular}

a The representative isolates used to analyze beta-tubulin gene sequences.

${ }^{\mathrm{b}}$ Carbendazim phenotypes of the three Colletotrichum species. HR, MR, S, and IS represent highly resistant, moderately resistant, sensitive, and insensitive, respectively.

${ }^{\mathrm{c}}$ Deduced amino acid substitution in beta-tubulin. E198A represents a mutation in codon 198 in which glutamic acid is replaced by alanine. F200Y represents a mutation in codon 200 in which phenylalanine is replaced by tyrosine. complex collected from China to thiophanate-methyl has been as high as $223.78 \mu \mathrm{g} / \mathrm{ml}$ (Jin 2013). However, information on the sensitivity of $C$. acutatum complex isolates to MBCs is limited. Isolates of $C$. acutatum from citrus have been found tolerant to $\mathrm{MBC}$ fungicides, and colonies were not smaller than those of a control of benomyl at $1,000 \mu \mathrm{l} / \mathrm{ml}$ (Peres et al. 2004). C. acutatum that grew on fungicideamended media at $100 \mu \mathrm{g} / \mathrm{ml}$ has shown insensitivity rather than resistance to thiophanate-methyl (Baggio et al. 2018). Similarly, all isolates of $C$. nymphaeae were insensitive to carbendazim in the present study. Mycelial growth of C. nymphaeae isolates was not inhibited completely on PDA containing $500 \mu \mathrm{g} / \mathrm{ml}$ of carbendazim.

Resistant isolates of $C$. siamense and C. fructicola had point mutations at codons 198 or 200 in TUB2. However, none of the sequenced isolates of $C$. nymphaeae had the most common mutations, similar to other reports on $C$. acutatum belonging to the C. acutatum complex (Baggio et al. 2018; Peres et al. 2004). Resistance in $C$. acutatum may be related to other mechanisms. Nakaune and Nakano (2007) reported that isolate of C. acutatum resistance to benomyl was not associated with codon mutation but caused by increased expression of CaTUB1 regulated by the protein CaBEN1. However, the mechanism of increasing gene expression is still unclear. According to the results of fungicide sensitivity assays that used carbendazim and sequence analyses of $T U B 2$, insensitivity to carbendazim may be a feature of $C$. nymphaeae.

It is interesting that the resistance phenotype caused by codon mutation was correlated with aggressiveness by contrasting the disease index with resistance codons of the three species in the present study. Most HR isolates of $C$. fructicola to carbendazim with E198A were the most aggressive to crowns, and the disease index was 91.7. Most MR isolates of $C$. siamense with F200Y had the strongest aggressiveness to leaves and fruit, and the disease index and lesion size were 93.6 and $20.0 \mathrm{~mm}$, respectively. All IS isolates of $C$. nymphaeae without common mutations in TUB2 were weakly aggressive to fruit, leaves, or crowns. However, more isolates should be tested for their aggressiveness and sequenced to explore this correlation.

Chemical application is necessary for controlling strawberry anthracnose. According to our study, isolates of $C$. siamense and C. fructicola from different strawberry parts showed a high frequency of resistance to carbendazim, and isolates of $C$. nymphaeae showed insensitivity to carbendazim. This finding suggests that carbendazim is an ineffective treatment for strawberry anthracnose and should not be considered for controlling anthracnose in strawberry production.

\section{Literature Cited}

Baggio, J. S., Wang, N. Y., Peres, N. A., and Amorim, L. 2018. Baseline sensitivity of Colletotrichum acutatum isolates from Brazilian strawberry fields to azoxystrobin, difenoconazole, and thiophanate-methyl. Trop. Plant Pathol. 43:533-542.

Carbone, I., and Kohn, L. M. 1999. A method for designing primer sets for speciation studies in filamentous ascomycetes. Mycologia 91:553-556.

Chung, W. H., Chung, W. C., Peng, M. T., Yang, H. R., and Huang, J. W. 2010. Specific detection of benzimidazole resistance in Colletotrichum gloeosporioides from fruit crops by PCR-RFLP. N. Biotechnol. 27:17-24.

Crous, P. W., Groenewald, J. Z., Risede, J. M., Simoneau, P., and Hyweljones, N. L. 2004. Calonectria species and their Cylindrocladium anamorphs: species with sphaeropedunculate vesicles. Stud. Mycol. 50:415-430.

Damm, U., Cannon, P. F., Woudenberg, J. H. C., and Crous, P. W. 2012. The Colletotrichum acutatum species complex. Stud. Mycol. 73:37-113.

Damm, U., Mostert, L., Crous, P. W., and Fourie, P. H. 2008. Novel Phaeoacremonium species associated with necrotic wood of Prunus trees. Persoonia 20:87-102.

Davidse, L. C. 1986. Benzimidazole fungicides: mechanism of action and biological impact. Annu. Rev. Phytopathol. 24:43-65.

Glass, N. L., and Donaldson, G. C. 1995. Development of primer sets designed for use with PCR to amplify conserved genes from filamentous ascomycetes. Appl. Environ. Microbiol. 61:1323-1330.

Guerber, J. C., Liu, B., Correll, J. C., and Johnston, P. R. 2003. Characterization of diversity in Colletotrichum acutatum sensu lato by sequence analysis of two gene introns, mtDNA and intron RFLPs, and mating compatibility. Mycologia 95: 872-895.

Hall, T. A. 1999. BioEdit: a user-friendly biological sequence alignment editor and analysis program for Windows 95/98/NT. Nucleic Acids Symp. 41:95-98.

Han, Y. C., Zeng, X. G., Xiang, F. Y., Ren, L., Chen, F. Y., and Gu, Y. C. 2016. Distribution and characteristics of Colletotrichum spp. associated with anthracnose of strawberry in Hubei, China. Plant Dis. 100:996-1006. 
Han, Y. C., Zeng, X. G., Xiang, F. Y., Zhang, Q. H., Guo, C., Chen, F. Y., and Gu, Y. C. 2018. Carbendazim sensitivity in populations of Colletotrichum gloeosporioides complex infecting strawberry and yams in Hubei Province of China. J. Integr. Agric. 17:1391-1400.

He, L. F., Li, X. X., Gao, Y. Y., Li, B. X., Mu, W., and Liu, F. 2019. Characterization and fungicide sensitivity of Colletotrichum spp. from different hosts in Shandong, China. Plant Dis. 103:34-43.

$\mathrm{Hu}$, S. 1990. The preliminary investigation of strawberry diseases. Collected abstracts for Fifth Meeting of East China, Chinese Society for Plant Pathology, Jiangsu 58-59.

Huang, J. K., and Zhang, G. Z. 2014. A method for inducing sporulation of Colletotrichum pathogen from strawberry. Plant Prot. 40:107-111.

Hyde, K. D., Cai, L., Cannon, P. F., Crouch, J. A., Crous, P. W., Damm, U., et al. 2009. Colletotrichum - names in current use. Fungal Divers. 39:147-182.

Jayawardena, R. S., Huang, J. K., Jin, B. C., Yan, J. Y., Li, X. H., Hyde, K. D., Bahkali, A. H., Yin, S. L., and Zhang, G. Z. 2016. An account of Colletotrichum species associated with strawberry anthracnose in China based on morphology and molecular data. Mycosphere 7:1147-1163.

Jin, B. C. 2013. Identification of pathogens causing strawberry anthracnose and fungicides screening for the pathogens. M.S. thesis. China Agricultural University, Beijing.

Johnston, P. R. 2000. The importance of phylogeny in understanding host relationships within Colletotrichum. Pages 21-28 in: Colletotrichum: host specificity, pathogenicity, and host-pathogen interactions. D. Prusky, M. B. Dickman, and S. Freeman, eds. APS Press, St Paul, MN.

Johnston, P. R., and Jones, D. 1997. Relationships among Colletotrichum isolates from fruit rots assessed using rDNA sequences. Mycologia 89:420-430.

Kuraku, S., Zmasek, C. M., Nishimura, O., and Katoh, K. 2013. aLeaves facilitates ondemand exploration of metazoan gene family trees on MAFFT sequence alignment server with enhanced interactivity. Nucleic Acids Res. 41 (W1):W22-W28.

Lin, T., Xu, X. F., Dai, D. J., Shi, H. J., Wang, H. D., and Zhang, C. Q. 2016. Differentiation in development of benzimidazole resistance in Colletotrichum gloeosporioides complex populations from strawberry and grape hosts. Australas. Plant Pathol. 45:241-249.

Liu, F., Wang, M., Damm, U., Crous, P. W., and Cai, L. 2016. Species boundaries in plant pathogenic fungi: a Colletotrichum case study. BMC Evol. Biol. 16:81.

Ministry of Agriculture of the People's Republic of China. 2018. The sown area and yield of vegetables, watermelons, muskmelons, strawberries and potatoes all over the country in 2016. China Vegetables 2018:18.

Nakaune, R., and Nakano, M. 2007. Benomyl resistance of Colletotrichum acutatum is caused by enhanced expression of $\beta$-tubulin 1 gene regulated by putative leucine zipper protein CaBEN1. Fungal Genet. Biol. 44:1324-1335.
Nalumpang, S., Miyamoto, Y., Miyake, C., Izumi, Y., Akitmitsu, K., and Kongtragoul, P. 2010. Point mutations in the beta-tubulin gene conferred carbendazim-resistant phenotypes of Colletotrichum gloeosporioides causing 'Nam Dok Mai' mango anthracnose. Int. J. Agric. Technol. 6: 365-378.

Nylander, J. A. A. 2004. MrModeltest v2. Program distributed by the author Evolutionary Biology Centre, Uppsala University,Uppsala, Sweden.

O’Donnell, K., and Cigelnik, E. 1997. Two divergent intragenomic rDNA ITS2 types within a monophyletic lineage of the fungus Fusarium are nonorthologous. Mol. Phylogenet. Evol. 7:103-116.

Peres, N. A. R., Souza, N. L., Peever, T. L., and Timmer, L. W. 2004. Benomyl sensitivity of isolates of Colletotrichum acutatum and C. gloeosporioides from citrus. Plant Dis. 88:125-130.

Ren, H. Y., Jiang, G. H., Fang, L., Zhang, Y. C., Yang, X. F., Miao, L. X., and Wang, H. R. 2011. Identification and characterization of the pathogen of strawberry anthracnose in Zhejiang Province. Acta Agriculturae Zhejiangensis 23:937-941.

Ren, X. J., Liang, Y., Lu, J. P., Yang, B. R., Xu, J. Y., and Dai, F. M. 2008 Identification of Colletotrichum species from strawberry in Shanghai. Acta Phytopathol. Sin. 38:325-328.

Ronquist, F., Teslenko, M., van der Mark, P., Ayres, D. L., Darling, A., Höhna, S., Larget, B., Liu, L., Suchard, M. A., and Huelsenbeck, J. P. 2012. MrBayes 3.2. efficient Bayesian phylogenetic inference and model choice across a large model space. Syst. Biol. 61:539-542.

Wang, F., Ma, Y., Gao, X. Y., and Zhang, Z. H. 2008. Study on the identification techniques for determining strawberry cultivar's resistance to anthracnose. J. Fruit Sci. 25:542-547.

Weir, B. S., Johnston, P. R., and Damm, U. 2012. The Colletotrichum gloeosporioides species complex. Stud. Mycol. 73:115-180.

White, T. J., Bruns, T., Lee, S., and Taylor, J. W. 1990. Amplification and direct sequencing of fungal ribosomal RNA genes for phylogenetics. Pages 315-322 in: PCR protocols: a guide to methods and applications. M. A. Innis, D. H. Gelfand, J. J. Sninsky, and T. J. White, eds. Academic Press, San Diego, CA.

Zhan, J. S., Wu, J. E., Liu, X. L., and Chen, F. P. 2014. Molecular basis of resistance of Phytopathogenic Fungi to several site-specific fungicides. Scientia Agricultura Sinica. 47:3392-3404.

Zhang, G. Z. 2015. Urgent attention to anthracnose root rot of strawberry is highly needed in China. Plant Prot. 41:234-236.

Zhang, Y. T., Wang, G. X., Chang, L. L., Dong, J., Zhong, C. F., and Wang, L. N. 2014. Current status of strawberry production and research in China. Acta Hortic. 1049:67-71. 